\title{
Grapado quirúrgico en la cirugía de control de daños del paciente gravemente traumatizado.
}

Revisión

Luis M. García-Núñez ${ }^{1}$, Lorena González², Ruy Cabello-Pasini³ ${ }^{3}$ Ignacio J. Magaña-Sánchez³ Javier Pérez-Aguirre ${ }^{3}$.

${ }^{1}$ Trauma Surgery and Surgical Critical Care, Department of Surgery - Division of Trauma, University of Medicine and Dentistry of New Jersey at Newark, ${ }^{2}$ University of Medicine and Dentistry of New Jersey at Newark, Newark, New Jersey, US. ${ }^{3}$ Sala de Cirugía de Hombres, Departamento de Cirugía General, ${ }^{3}$ Hospital Central Militar, Secretaría de la Defensa Nacional, ${ }^{3}$ Servicio de Colon y Recto, Departamento de Cirugía General, Hospital Central Militar, Secretaría de la Defensa Nacional, México, Distrito Federal, México.

\section{RESUMEN.}

Introducción. Los dispositivos de grapado quirúrgico (DGQ) han demostrado su utilidad en la cirugía de control de daños (CCD), dado que disminuyen el tiempo en el que el paciente se encuentra en el salón de operaciones, y por tanto favorecen su pronto traslado a la Unidad de Cuidados Intensivos, para iniciar el manejo para su recuperación metabólica.

Fuentes. Búsqueda bibliográfica y electrónica en la red de información referente al empleo de instrumentos de grapado quirúrgico en la cirugía del control de daños en el paciente politraumatizado, así como imágenes clínicas y recomendaciones de la propia experiencia de los autores.

Resultados. En este artículo mostramos diferentes estrategias quirúrgicas de control de daños aplicadas a distintas áreas corporales del paciente gravemente traumatizado, utilizando DGQ.

Conclusiones. Los dispositivos de grapado quirúrgico disminuyen el tiempo de intervención, sin comprometer la estrategia quirúrgica, y son instrumentos adjuntos muy valiosos para la institución de la cirugía de control de daños en el paciente traumatizado.

(Rev Biomed 2006; 17:124-131)

Palabras clave: grapado quirúrgico, trauma, control de daños.

\section{SUMMARY.}

Surgical stapling in damage-control surgery of the critically ill trauma patient.

Introduction. Surgical stapling devices have demonstrated their usefulness in damage control surgery (DCS), given they decrease the length of time in which the patient stays in the operating room, and thus favoring his/her early transportation to the Intensive Care Unit for the recovery of his/ her former metabolic status.

Data-source. Web-electronic and bibliographic research of current information related to the use

Solicitud de sobretiros: Mayor Médico Cirujano Luis M. García-Núñez. Circuito Naranjeros No. 22, Colonia Club de Golf México, Tlalpan, Distrito Federal 14620, México. Teléfono 5573-0026, FAX: 5573-0026, Correo electrónico: luismanuelgarcianunez@yahoo.com, lmgarcian@hotmail.com Recibido el 23/Septiembre/2005. Aceptado para publicación el 14/Diciembre/2005. 
LM García-Núñez, L González, R Cabello-Pasini, IJ Magaña-Sánchez, J Pérez-Aguirre.

of surgical-stapling devices in damage control surgery of the trauma patient, and clinical images and recommendations from the authors' personal experience.

Results. In this paper, we show different operative strategies for damage control surgery in the critically ill trauma patient, by using surgical stapling devices.

Conclusions. Surgical-stapling devices decrease the length of surgical time, without impairment of the surgical strategy, and they are valuable auxilliary instruments for the institution of damage-control surgery in the trauma patient.

(Rev Biomed 2006; 17:124-131)

Key words: surgical stapling, trauma, damage control.

\section{INTRODUCCIÓN.}

Los dispositivos de grapado quirúrgico (DGQ) han disminuido notablemente el tiempo de estancia en el quirófano, dada su simplicidad de uso y rapidez de aplicación. En el área de la cirugía de trauma, los DGQ han revolucionado de manera radical la práctica de la cirugía de control de daños (CCD) del paciente críticamente lesionado. Según lo establecido por García-Núñez et al (1), en una extensa revisión de la literatura, uno de los principios fundamentales de la CCD establece que el tiempo operatorio debe abreviarse con el fin de trasladar al paciente a la Unidad de Cuidados Intensivos para dar paso a la corrección de las alteraciones metabólicas e interrumpir la progresión de la tríada letal de Burch (acidosis, hipotermia y coagulopatía) (2), y al mismo tiempo, apartarse de la posibilidad de que la tétrada de Asensio haga su aparición (complejo letal consistente en la tríada de Burch más disritmia cardíaca asociada), lo cual traduce un estado crítico y una alta probabilidad de mortalidad (3). Múltiples estudios en la literatura (Wagner et al (1996) (4), Brasel et al (2000) (5), Cothren et al (2002) (6) y Kirkpatrick et al (2003)(7)) han establecido que los instrumentos de grapado quirúrgico disminuyen el tiempo de intervención en la cirugía de control de daños, sin comprometer a la estrategia quirúrgica en sí.

¿En qué momento debe instituirse la práctica de la CCD?. Los conceptos varían de acuerdo a la fuente. Sin embargo, Asensio et al $(2,3)$, con base en un estudio retrospectivo de 548 pacientes con síndrome de exsanguinación, estableció que los diferentes estudios previos relacionados con la temática, no identifican la presentación clínica de los pacientes a su arribo al Departamento de Urgencias y proponen estrategias de abreviación de las maniobras quirúrgicas, una vez que el paciente manifiesta coagulopatía clínicamente patente, lo cual traduce un agotamiento metabólico claro y una alta mortalidad; lo mismo señala para los parámetros clásicos utilizados como marcadores de institución (volumen de transfusión, temperatura, $\mathrm{pH}$, pruebas de coagulación-TP, TTP -) (8-11). Por tanto, ha validado estadísticamente y recomienda la estrecha monitorización de los siguientes marcadores transoperatorios como indicadores de la institución de esta técnica, lo cual sin duda se simplifica y logra exitosamente en la mayoría de los casos con el uso de DGQ: 1) volumen de transfusión $>4,000 \mathrm{ml}$ de concentrado eritrocitario, 2) volúmen de transfusión $>5,000 \mathrm{ml}$ si se han usado de manera conjunta concentrados eritrocitarios y otros componentes sanguíneos, 3) volumen total infundido $>12,000 \mathrm{ml}$, si se han empleado cristaloides, coloides y productos sanguíneos, 4) $\mathrm{pH}>7.2,5$ ) temperatura $<34^{\circ} \mathrm{C}$, y 6 ) pérdida estimada de sangre de más de 5,000 ml.

\section{UTILIDAD DE LOS DGQ EN LOS DISTIN- TOS ESCENARIOS DE LA CIRUGÍA DE CONTROL DE DAÑOS DEL PACIENTE CRÍTICAMENTE LESIONADO.}

Tegumentos. Eventualmente, la institución de la CCD exige la intervención en tegumentos. La piel puede afrontarse con el uso de engrapadoras de piel multidireccionales (Skin Stapler ${ }^{\circledR}$; grapas regulares

\section{Revista Biomédica}


Grapado en cirugía de control de daños.

de $0.53 \mathrm{~mm}$ de diámetro, $5.7 \mathrm{~mm}$ de corona y brazos de $3.9 \mathrm{~mm}$; o anchas de $0.58 \mathrm{~mm}$ de diámetro, $6.9 \mathrm{~mm}$ de corona y brazos de $3.9 \mathrm{~mm}$ ).

Tórax. Una vez que se ha decidido la institución de la CCD en el tórax, las maniobras con posibilidad de practicarse son las siguientes: 1) resección pulmonar en cuña (no anatómica) grapada: lo cual se puede efectuar con engrapadoras lineares cortantes de 56 unidades (Proximate Linear Cutter TLC55 ${ }^{\circledR}$; grapas de $0.23 \mathrm{~mm}$ de diámetro, con corona de $3 \mathrm{~mm}$, brazos de $3.85 \mathrm{~mm}$, longitud de línea de grapado de $57 \mathrm{~mm}$ y longitud de línea de corte de $53 \mathrm{~mm}$ ); de 76 unidades (Proximate Linear Cutter TLC75 ${ }^{\circledR}$; grapas de $0.20 \mathrm{~mm}$ de diámetro, corona de $3 \mathrm{~mm}$, brazos de $3.85 \mathrm{~mm}$, longitud de línea de grapado de 77 mm y longitud de línea de corte de $73 \mathrm{~mm}$ ); o de 100 unidades (Proximate Linear Cutter TLC10 ${ }^{\circledR}$; grapas de 0.20 $\mathrm{mm}$ de diámetro, con corona de $3 \mathrm{~mm}$, brazos de $3.85 \mathrm{~mm}$, longitud de línea de grapado de $102 \mathrm{~mm}$ y longitud de línea de corte de $98 \mathrm{~mm}$ )(figura 1); 2) tractotomía pulmonar grapada con técnica de Asensio: dicha técnica (12) puede practicarse con engrapadoras lineares cortantes de 56 unidades (Proximate Linear Cutter TLC55 ${ }^{\circledR}$; grapas de 0.23 $\mathrm{mm}$ de diámetro, con corona de $3 \mathrm{~mm}$, brazos de $3.85 \mathrm{~mm}$, longitud de línea de grapado de $57 \mathrm{~mm}$

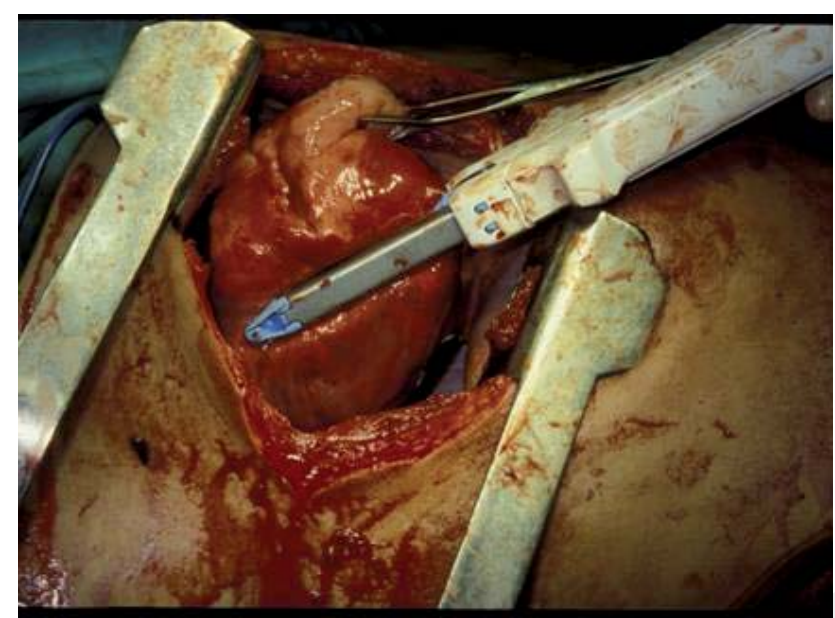

Figura 1.- Aplicación de la engrapadora para la resección pulmonar en cuña. Los segmentos a resecar se limitan por medio de la aplicación de dispositivos de grapado orientados en direcciones perpendiculares, tratando de mantener la mayor cantidad de tejido sano. y de línea de corte de $53 \mathrm{~mm}$ ) o de 76 unidades (Proximate Linear Cutter TLC75 ${ }^{\circledR}$; grapas de $0.2 \mathrm{~mm}$ de diámetro, corona de $3 \mathrm{~mm}$, brazos de $3.85 \mathrm{~mm}$, longitud de línea de grapado de $77 \mathrm{~mm}$ y de línea de corte de $73 \mathrm{~mm}$ )(figuras 2 a 4); 3) lobectomía anatómica grapada: independientemente de los detalles quirúrgicos que involucran a cada una de las 5 diferentes lobectomías posibles en este escenario, y por las cuales se remite al lector hacia los textos propios de la especialidad, existen recomendaciones claras con respecto al uso de engrapadoras en las lobectomías pulmonares. Asensio, en 2005, estableció genéricamente que los vasos sanguíneos

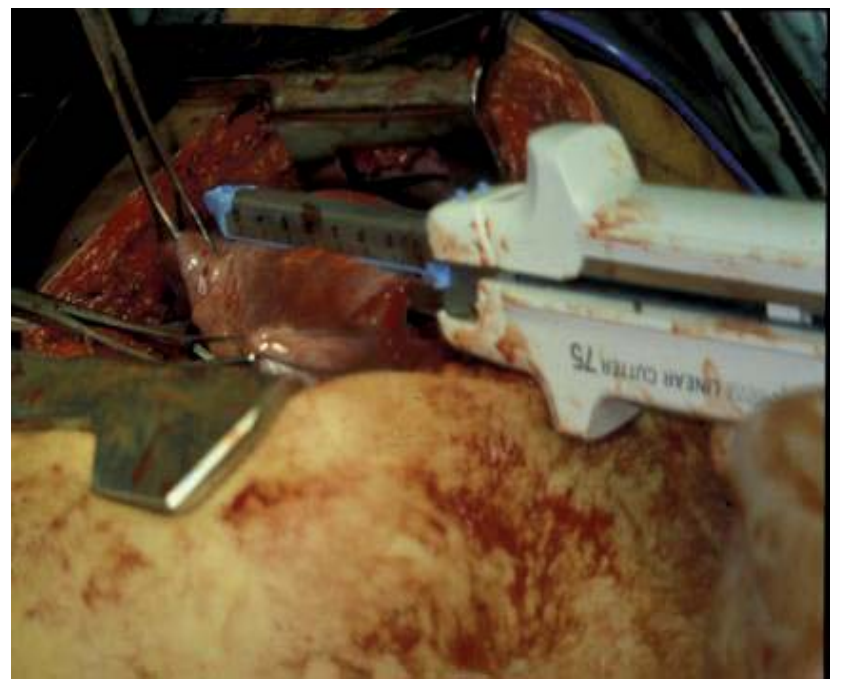

Figura 2.- Inserción de engrapadora lineal cortante a través de los orificios del tracto de la herida pulmonar penetrante.

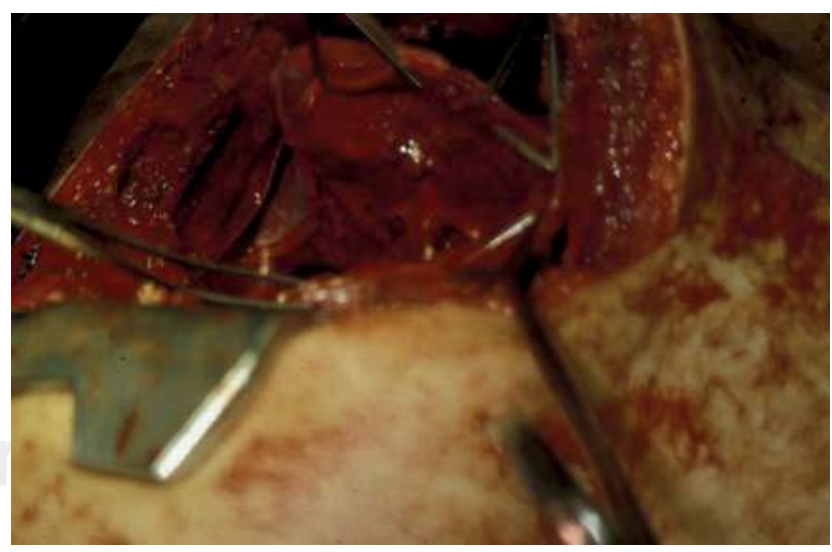

Figura 3.- Apertura del tracto de la herida pulmonar penetrante y ligadura de vasos sangrantes y vías aéreas distales con fuga aérea visible.

Vol. 17/No. 2/Abril-Junio, 2006 
LM García-Núñez, L González, R Cabello-Pasini, IJ Magaña-Sánchez, J Pérez-Aguirre.

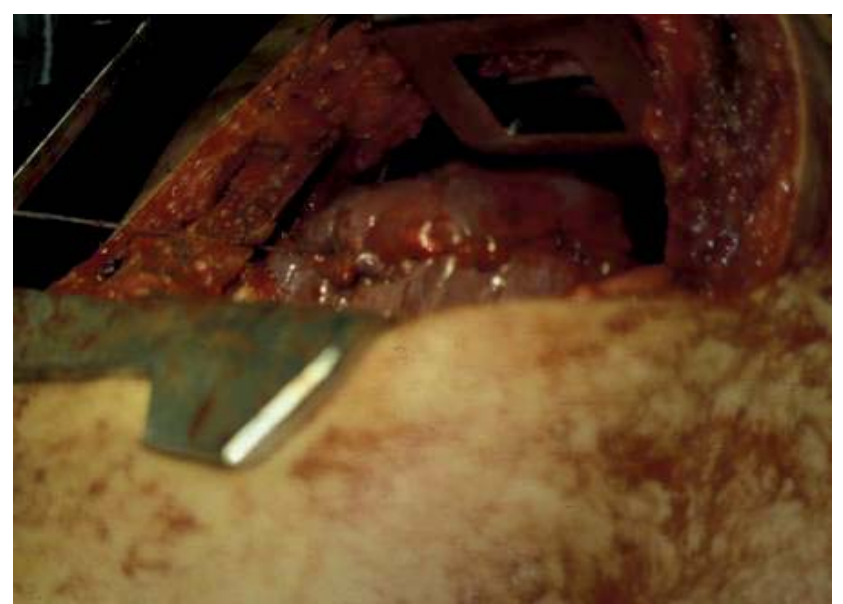

Figura 4.- Sutura del tracto con material absorbible (catgut crómico 3-0 o equivalente), manteniendo los orificios de entrada y salida abiertos, evitando así la posibilidad de embolia aérea.

pulmonares deben graparse por vía de alguno de los siguientes dispositivos (longitud de brazo de la grapa de $3.5 \mathrm{~mm}$ o menor): engrapadora lineal vascular de 15 unidades (Proximate RVLS $T L V 30^{\circledR}$; grapas de $0.2 \mathrm{~mm}$ de diámetro, corona de $4 \mathrm{~mm}$, brazos de $2.5 \mathrm{~mm}$, y longitud de línea de grapado de $30 \mathrm{~mm}$ ); engrapadora lineal vascular de 23 unidades (Proximate RVLS TX30V ${ }^{\circledR}$; triple línea de grapas de $0.2 \mathrm{~mm}$ de diámetro, corona de $3 \mathrm{~mm}$, brazos de $2.5 \mathrm{~mm}$ y longitud de línea de grapado de $30 \mathrm{~mm}$ ); engrapadora lineal de 19 unidades (Proximate RLS AX55B ${ }^{\circledR}$; grapas de 0.23 $\mathrm{mm}$ de diámetro, corona de $4 \mathrm{~mm}$, brazos de 3.5 $\mathrm{mm}$, longitud de línea de grapado de $55 \mathrm{~mm}$ ); o engrapadora lineal de 11 unidades (Proximate RLS TX30B ${ }^{\circledR}$; grapas de $0.23 \mathrm{~mm}$ de diámetro, corona de $4 \mathrm{~mm}$, brazos de $3.5 \mathrm{~mm}$, longitud de línea de grapado de $30 \mathrm{~mm}$ ). De la misma forma, Asensio nos recalca la necesidad de reforzar la línea de grapado con una sutura continua con monofilamento de polipropileno (4-0 a 6-0). Las estructuras bronquiales pueden graparse sin necesidad de reforzar (salvo en caso de fuga aérea residual) con una engrapadora lineal de 11 unidades (Proximate RLS TLH30 ${ }^{\circledR}$; grapas de 0.3 $\mathrm{mm}$ de diámetro, corona de $4 \mathrm{~mm}$, brazos de 4.8 $\mathrm{mm}$, longitud de línea de grapado de $30 \mathrm{~mm}$ ); o engrapadora lineal de 19 unidades (Proximate
AX55G ${ }^{\circledR}$; grapas de $0.28 \mathrm{~mm}$ de diámetro, corona de $4 \mathrm{~mm}$, brazos de $4.8 \mathrm{~mm}$, longitud de línea de grapado de $55 \mathrm{~mm}$ ). El parénquima pulmonar se secciona con las engrapadoras lineales cortantes recomendadas para la resección segmentaria o tractotomía grapadas; 4) neumonectomía grapada: la neumonectomía grapada puede efectuarse con auxilio de una engrapadora de 33 unidades (Proximate RLS TL90; grapas de $0.23 \mathrm{~mm}$ de diámetro, corona de $4 \mathrm{~mm}$, brazos de $4.5 \mathrm{~mm}$, longitud de línea de grapado de 90 $\mathrm{mm}$ ), asegurando tanto las estructuras bronquiales como vasculares en continuidad, con una sola línea de grapas (figuras 5 y 6); 5) ligadura grapada de estructuras torácicas - conducto torácico, vena ázigos o hemiázigos: las estructuras vasculares menores y el conducto torácico se pueden ligar por medio dispositivos de grapado individual: Ligaclip ${ }^{\circledR}$ MCA, Multiple Clip Applier; 6) corazón: a pesar de que Mayrose et al (13) han reportado que el engrapado del miocardio provee de igual resistencia y características mecánicas que la sutura manual con monofilamento sintético en las heridas cardiacas penetrantes, Asensio no recomienda practicar esta maniobra, dada la relativa friabilidad del músculo cardiaco.

Abdomen. Es notable la variedad de técnicas que pueden practicarse en el abdomen con los DGQ.

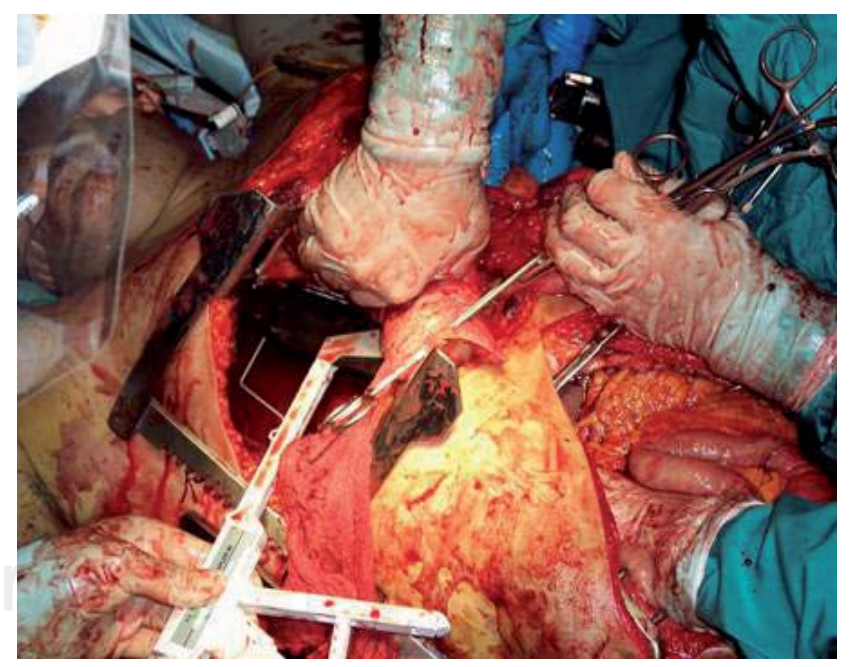

Figura 5.- Aplicación de un DGQ lineal en el hilio pulmonar, durante una Toracotomía en el Salón de Operaciones. El pulmón derecho se retrajo cefálicamente de forma manual.

\section{Revista Biomédica}


Las más comúnmente requeridas en la CCD abdominal son las siguientes:

Tracto gastrointestinal: En este escenario, es posible resecar segmentos intestinales, dejándolos en descontinuidad, para reestablecer la continuidad del tránsito en una cirugía ulterior. Para este fin, el instrumento preferido es una engrapadora lineal cortante de 76 unidades (Proximate Linear Cutter TLC75 ${ }^{\circledR}$; grapas de 0.2 $\mathrm{mm}$ de diámetro, corona de $3 \mathrm{~mm}$, brazos de 3.85 $\mathrm{mm}$, longitud de línea de grapado de $77 \mathrm{~mm}$ y de línea de corte de 73 mm)(figuras 7 y 8). También es posible construir anastomosis intestinales laterolaterales con este mismo DGQ. No obstante, en

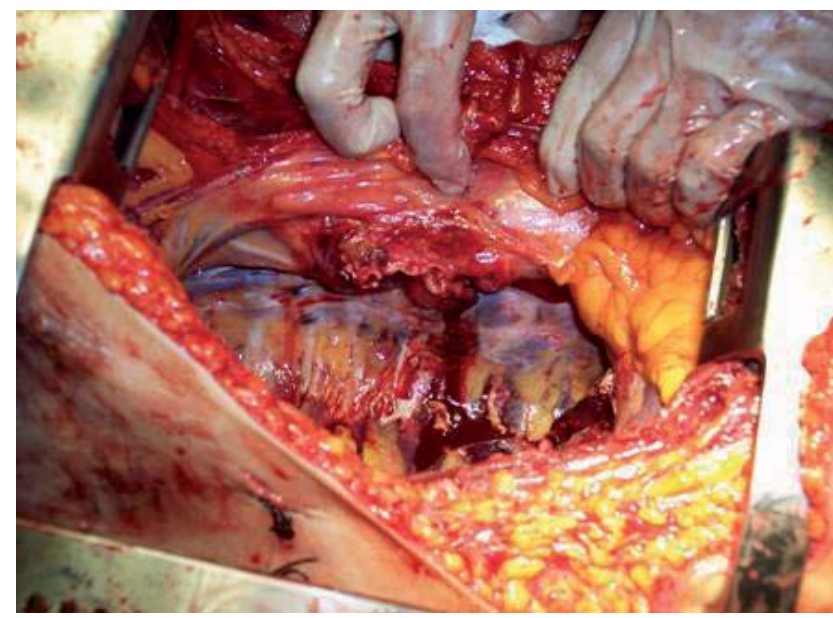

Figura 6.- Fotografía del mismo paciente, en donde se observa la línea de grapas en el hilio pulmonar derecho.

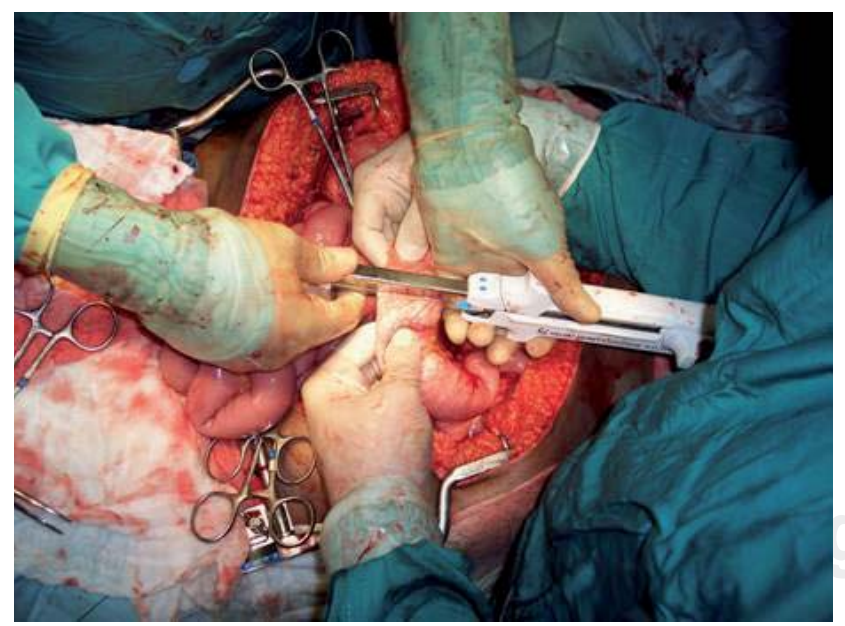

Figura 7.- Aplicación de una engrapadora lineal cortante para resecar un segmento intestinal desvitalizado. El tracto gastrointestinal se dejó en discontinuidad.

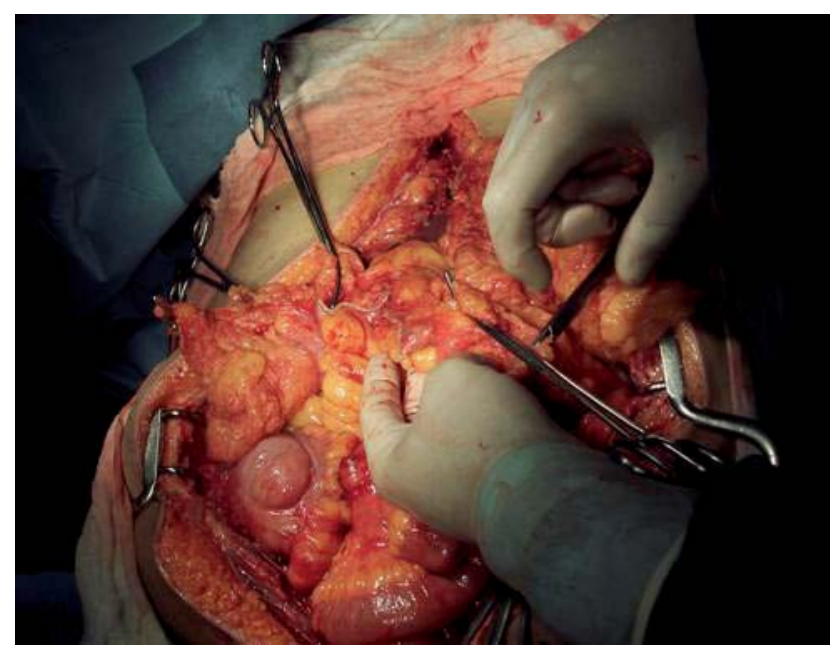

Figura 8.- Imágen en la cual se demuestra la descontinuidad del tracto gastrointestinal, tras el grapado y resección del asa intestinal lesionada.

virtud de las características de la técnica, esto último no forma parte habitual de la práctica de la CCD. Los pedículos vasculares mayores pueden seccionarse con las engrapadoras vasculares recomendadas para el aseguramiento y sección de los vasos pulmonares. Los vasos individuales y pedículos vasculares menores pueden asegurarse con el uso de Ligaclip ${ }^{\circledR}$ MCA, Multiple Clip Applier.

Tracto genitourinario: Las lesiones hiliares renales pueden ligarse de forma rápida y segura manualmente; la ligadura individual de la arteria y vena renales permanece en pie como la técnica de elección para el control de hemorragia debida a lesiones hiliares. Sin embargo, Rapp y Salhvav (2004) han reportado la confiabilidad del aseguramiento y sección en continuidad del hilio renal en casos de sangrado masivo. Esto puede lograrse por medio de los siguientes dispositivos: engrapadora lineal vascular de 15 unidades (Proximate RVLS TLV30 ${ }^{\circledR}$; grapas de $0.2 \mathrm{~mm}$ de diámetro, corona de $4 \mathrm{~mm}$, brazos de $2.5 \mathrm{~mm}$, y longitud de línea de grapado de 30 $\mathrm{mm}$ ); engrapadora lineal vascular de 23 unidades (Proximate RVLS TX30V ${ }^{\circledR}$; triple línea de grapas de $0.2 \mathrm{~mm}$ de diámetro, corona de $3 \mathrm{~mm}$, brazos de $2.5 \mathrm{~mm}$ y longitud de línea de grapado de $30 \mathrm{~mm}$ ); engrapadora lineal de 19 unidades (Proximate RLS

Vol. 17/No. 2/Abril-Junio, 2006 


\section{LM García-Núñez, L González, R Cabello-Pasini, IJ Magaña-Sánchez, J Pérez-Aguirre.}

AX55B ${ }^{\circledR}$; grapas de $0.23 \mathrm{~mm}$ de diámetro, corona de $4 \mathrm{~mm}$, brazos de $3.5 \mathrm{~mm}$, longitud de línea de grapado de $55 \mathrm{~mm}) ;$ o engrapadora lineal de 11 unidades (Proximate RLS TX30B ${ }^{\circledR}$; grapas de 0.23 $\mathrm{mm}$ de diámetro, corona de $4 \mathrm{~mm}$, brazos de 3.5 $\mathrm{mm}$, longitud de línea de grapado de $30 \mathrm{~mm}$ ). No hay reportes validados del uso de engrapadoras para la ligadura del uretero o intervenciones sobre el parénquima renal.

Hígado: En ocasiones se requieren resecciones en cuña del parénquima hepático cuando este se encuentra desvitalizado. Kaneko et al (14) han propuesto el uso de engrapadoras lineales para la sección del parénquima hepático. Estos dispositivos deben colocar líneas de grapado de 35 a $45 \mathrm{~mm}$ de longitud; no especifican la medida de la corona pero establecen que los brazos de la grapa deben medir $3.5 \mathrm{~mm}$. Los vasos sanguíneos y los ductos biliares pueden asegurarse individualmente con el uso del siguiente dispositivo: Ligaclip ${ }^{\circledR}$ MCA, Multiple Clip Applier)(figura 9).

Páncreas: En su clásica revisión, Asensio (15) establece genéricamente que para la resección pancreática durante la CCD, cuando esta se encuentra indicada (lo cual sucede muy raramente), puede emplearse el siguiente dispositivo: engrapadora lineal de 19 unidades

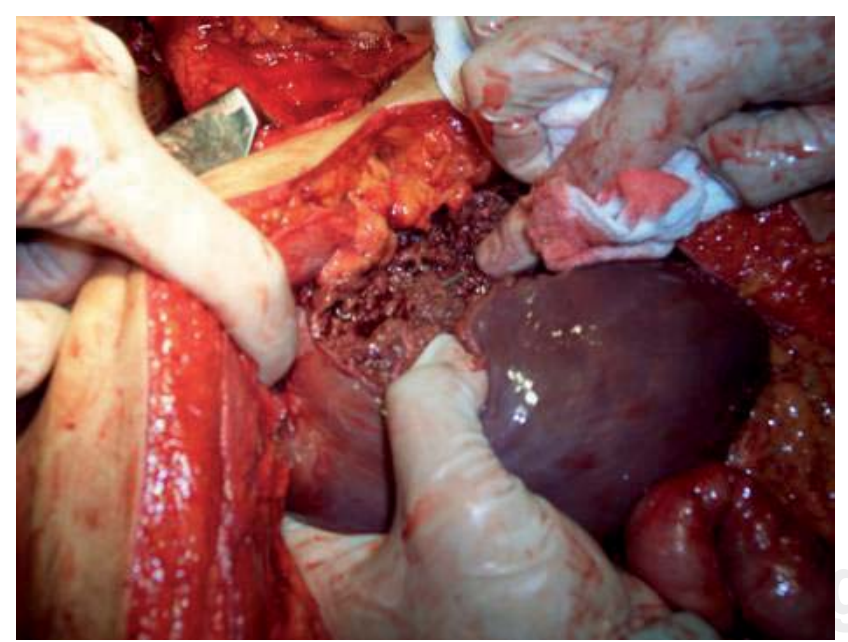

Figura 9.- Grapa metálica aplicada con Ligaclip ${ }^{\circledR} M C A$, Multiple Clip Applier (Ethicon Endosurgery, Somerville, NJ, US) en un vaso sanguíneo individual en el interior del parénquima hepático, durante una técnica de CCD.

\section{Revista Biomédica}

(Proximate RLS AX55B ${ }^{\circledR}$; grapas de $0.23 \mathrm{~mm}$ de diámetro, corona de $4 \mathrm{~mm}$, brazos de $3.5 \mathrm{~mm}$, longitud de línea de grapado de $55 \mathrm{~mm}$ ).

Bazo: El hilio esplénico puede seccionarse y las estructuras vasculares asegurarse por medio de alguno de los siguientes dispositivos: engrapadora lineal vascular de 15 unidades (Proximate RVLS $T L V 30^{\circledR}$; grapas de $0.2 \mathrm{~mm}$ de diámetro, corona de $4 \mathrm{~mm}$, brazos de $2.5 \mathrm{~mm}$, y longitud de línea de grapado de $30 \mathrm{~mm}$ ); engrapadora lineal vascular de 23 unidades (Proximate RVLS TX30V $V^{\circledR}$; triple línea de grapas de $0.2 \mathrm{~mm}$ de diámetro, corona de $3 \mathrm{~mm}$, brazos de $2.5 \mathrm{~mm}$ y longitud de línea de grapado de $30 \mathrm{~mm}$ ); engrapadora lineal de 19 unidades (Proximate RLS AX55B ${ }^{\circledR}$; grapas de 0.23 $\mathrm{mm}$ de diámetro, corona de $4 \mathrm{~mm}$, brazos de 3.5 $\mathrm{mm}$, longitud de línea de grapado de $55 \mathrm{~mm}$ ); o engrapadora lineal de 11 unidades (Proximate RLS $T X 30 B^{\circledR}$; grapas de $0.23 \mathrm{~mm}$ de diámetro, corona de $4 \mathrm{~mm}$, brazos de $3.5 \mathrm{~mm}$, longitud de línea de grapado de $30 \mathrm{~mm}$ ). Los vasos individuales pueden asegurarse por medio del dispositivo Ligaclip ${ }^{\circledR}$ MCA, Multiple Clip Applier. No hay suficiente experiencia para recomendar la esplenectomía segmentaria grapada en la CCD.

Aplicación de cierre protésico de la pared abdominal: Durante la CCD aplicada al abdomen, es común efectuar el cierre protésico de la pared abdominal, pues los pacientes pueden requerir intervenciones seriadas (16). La bolsa plástica de solución intravenosa que cubre las vísceras puede sujetarse a los bordes cutáneos fácil y rápidamente empleando engrapadoras de piel multidireccionales (Skin Stapler ${ }^{\circledR}$; grapas regulares de $0.53 \mathrm{~mm}$ de diámetro, $5.7 \mathrm{~mm}$ de corona y brazos de $3.9 \mathrm{~mm}$; o anchas de $0.58 \mathrm{~mm}$ de diámetro, $6.9 \mathrm{~mm}$ de corona y brazos de $3.9 \mathrm{~mm}$ )(figura 10).

Extremidades. La CCD se practica ocasionalmente en las extremidades. El escenario clínico más común en el cual el cirujano de trauma usa DGQ es el síndrome compartimental. En estos pacientes, se requieren amplias fasciotomías y protección de las heridas con apósitos biológicos o sintéticos, o colocación de dispositivos de cierre 
Grapado en cirugía de control de daños.

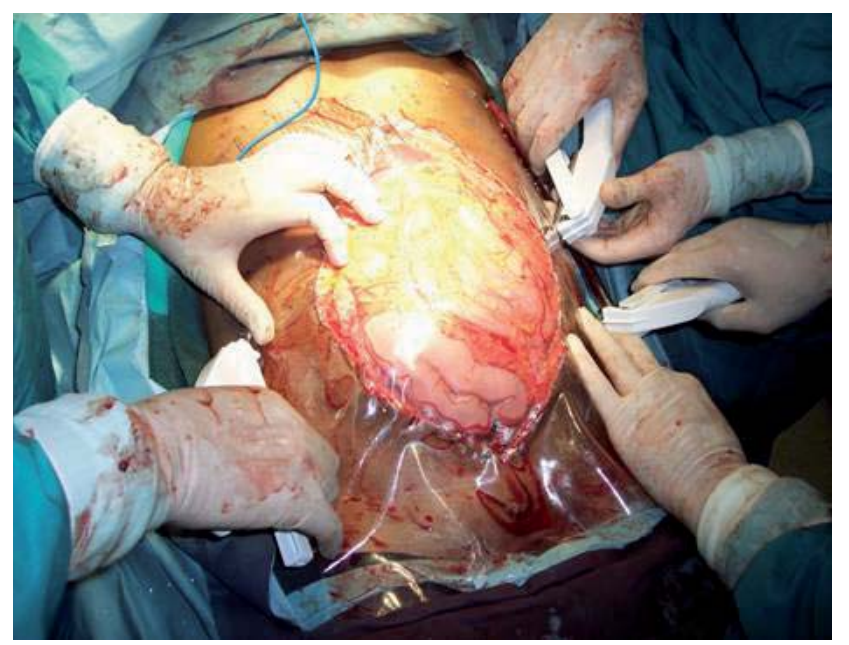

Figura 10.- Aplicación de la prótesis de la pared abdominal (bolsa plástica de solución intravenosa), asegurándola a la piel con engrapadoras apropiadas.

de herida asistida por succión (V.A.C. Vaccum Assisted Closure ${ }^{\circledR}$, KCI Therapies, US))(figura 11). Usualmente, se emplean engrapadoras de piel multidireccionales con las características previamente mencionadas en el apartado de tegumentos.

\section{DISCUSIÓN.}

Los DGQ han impactado de forma dramática la práctica de la CCD en el paciente críticamente lesionado. Existen múltiples estudios (4-8), tanto en el área de la cirugía de trauma como

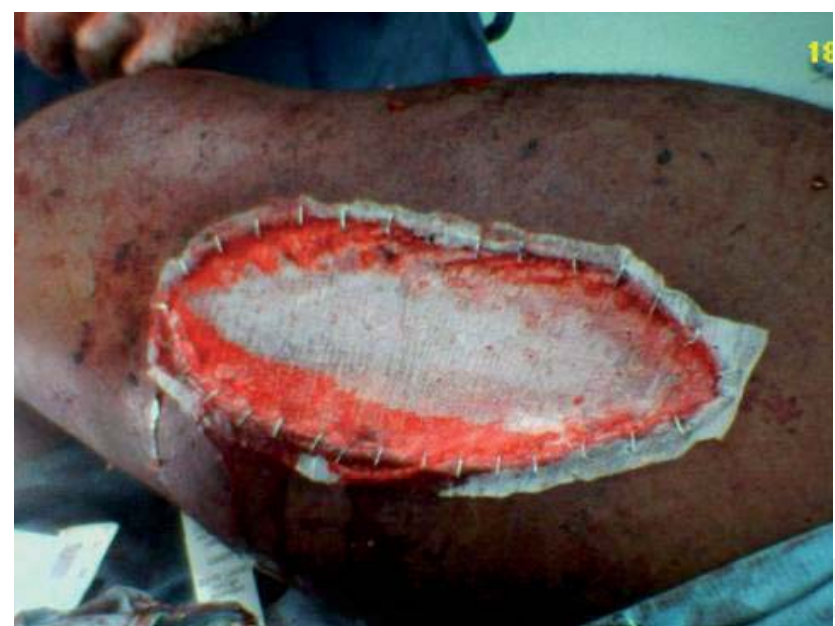

Figura 11.- Colocación de apósito sintético (Biobrane ${ }^{\circledR}$, Bertek Pharmaceuticals, Morgantown, WVA, US) en una herida de fasciotomía, asegurado por medio de grapas de piel. en algunas otras especialidades quirúrgicas, en los cuales se ha verificado que las maniobras quirúrgicas efectuadas por medio de instrumentos de grapado cumplen con los mismos objetivos de función que las técnicas construídas manualmente, sin sacrificio de la seguridad, y beneficiando con un obvio ahorro de tiempo a la intervención.

\section{CONCLUSIONES.}

El uso de DGQ favorece uno de los objetivos cardinales de la institución de la CCD, pues las maniobras quirúrgicas que pueden ser efectuadas con estos instrumentos conservan los principios de estructuración y cumplen con los mismos objetivos que aquellas construidas manualmente (4-8), con la gran ventaja de que acortan el tiempo en el cual el paciente se encuentra sometido a intervención quirúrgica. Esto se traduce en un pronto traslado a la Unidad de Cuidados Intensivos para la restauración de su estado metabólico.

${ }^{\circledR}$ Ethicon Endosurgery, Somerville, NJ, US. El autor principal hace extensa referencia a estos instrumentos, dado que su experiencia está basada principalmente en el conocimiento y uso de estos dispositivos en específico. No atiende a ningún interés comercial o particular.

\section{REFERENCIAS.}

1.- García-Núñez LM, Cabello PR, Moreno DLF, et al. Conceptos actuales en Cirugía de Trauma: El control de daños no termina en el quirófano. Observaciones no publicadas, Revista “Trauma, la Urgencia Médica de Hoy”; Asociación Mexicana de Medicina y Cirugía del Trauma. México, 2005.

2.- Asensio JA, McDuffie L, Petrone P, et al. Reliable variables in the exsanguinated patient which indicate damage control and predict outcome. Am J Surg 2001;182:743-51.

3.- Asensio JA, Petrone P, O’Shanahan G, Kuncir E. Managing exsanguination: what we know about damage control/bail out is not enough. BUMC Proceedings 2003;16:294-6.

4.- Wagner J, Obeid FN, Karmy-Jones RC, Casey G, Sorensen VJ, Horst HM. Trauma pneumonectomy revisited: 


\section{LM García-Núñez, L González, R Cabello-Pasini, IJ Magaña-Sánchez, J Pérez-Aguirre.}

the role of simultaneously stapled pneumonectomy. J Trauma 1996;40:590-4.

5.- Brasel KJ, Weiselt JA. Damage control in trauma surgery. Curr Opin Crit Care 2000;6:276-80.

6.- Cothren C, Moore EE, Biffl WL, Franciose R, Offner $\mathrm{P}$, Burch JM. Lung-sparing techniques are associated with improved outcome compared with anatomic resection for severe lung injuries. J Trauma 2002;53:483-7.

7.- Kirkpatrick AW, Baxter KA, Simons RK, Germann E, Lucas CE, Ledgerwood AM. Intra-abdominal complications after surgical repair of small bowel injuries: an International review. J Trauma 2003;55:399-406.

8.- Lynn M, Jeroukhimov I, Klein Y, Martinowitz U. Updates in the management of severe coagulopathy in trauma patients. Intensive Care Med 2002;28(Suplement):S241-7.

9.- Hirshberg A, Dugas M, Banez EI, et al. Minimizing dilutional coagulopathy in exsanguinating hemorrhage: a computer simulation. J Trauma 2003;54:454-63.

10.- Cosgriff N, Moore EE, Sauaia A, et al. Predicting life threatening coagulopathy in the massively transfused trauma patient: hypothermia and acidosis revisited. J Trauma 1997;42:857-62.

11.- García-Núñez LM, Padilla SR, Lever RCD, et al. Sugerencias acerca de los objetivos finales en la resuscitación del paciente exsanguinado por trauma. Siempre es bueno saber a donde ir. Observaciones no publicadas, Revista “Trauma, la Urgencia Medica de Hoy”, México, 2005.

12.- Asensio JA, Demetriades D, Berne JD, et al. Stapled pulmonary tractotomy: a rapid way to control hemorrhage in penetrating pulmonary injuries. J Am Coll Surg 1997;185: 486-7.

13.- Mayrose JM, Jehle D, Moscati R, Lerner EB, Abrams B. Comparison of staples versus sutures in the repair of penetrating cardiac wounds. J Trauma 1999;46:441-3.

14.- Kaneko H, Otsuka Y, Takagi S, Tsuchiya M, Tamura A, Shiba T. Hepatic resection using stapling devices. Am J Surg 2004;187:280-4.
15.- Asensio JA, Demetriades D, Hanpeter DE, Gambaro E, Chahwan S. Management of pancreatic injuries. Curr Prob Surg 1999;36:325-419.

16.- García-Núñez LM, Magaña SIJ, Noyola VHF, Belmonte MC, Rosales ME. Manejo con técnica de abdomen abierto en pacientes críticos. Experiencia de dos años en el Hospital Central Militar. Rev Sanid Milit Mex 2003;57:232-6.

\section{Revista Biomédica}

